\title{
MODELING OF THERMAL CONTACT THROUGH GAP WITH THE USE OF FINITE ELEMENT METHOD
}

\author{
Ewa Wegrzyn-Skrzypczak ${ }^{1}$, Tomasz Skrzypczak ${ }^{2}$ \\ ${ }^{1}$ Institute of Mathematics, Czestochowa University of Technology \\ Częstochowa, Poland \\ ${ }^{2}$ Institute of Mechanics and Machine Design Fundamentals \\ Czestochowa University of Technology \\ Czestochowa, Poland \\ ewa.skrzypczak@im.pcz.pl,skrzyp@imipkm.pcz.pl
}

\begin{abstract}
In the paper the mathematical and numerical descriptions of the general case of thermal contact between two flat bodies are presented. The numerical model of the problem is based on the Finite Element Method (FEM). Variable width of the contact gap between interacting bodies is considered. The model allows the use of independent spatial discretization of the contacting components, which means that the edges of the finite elements lying on the both sides of the contact gap need not be matched. The algorithm of treatment of the fourth kind boundary condition is described in details.
\end{abstract}

Keywords: computational mechanics, thermal contact problem, finite element method

\section{Introduction}

Machine parts that work together are in mechanical and thermal contact. Mechanical contact is connected with the direct interaction of the surfaces of working elements. Thermal contact does not require direct contact because the heat transfer between the elements can be carried out through a gap. The mathematical description of the thermal contact is to supplement the heat conduction equation by the fourth type of boundary condition, also called the contact condition. One can distinguish two variants of this condition describing the so-called perfect contact and the contact via a gap, the width of which in the general case can be varied. Descriptions of the problem of thermal contact between non-ideal surfaces can be found in the literature dating back more than 50 years [1]. The numerical solution of transient heat transfer between two semi-infinite flat bodies using the Finite Difference Method (FDM) was presented in [2]. Analytical solutions of the problem using the Fourier series was presented in [3]. Currently the ideal and non-ideal thermal contact is the subject of interest of many scientists. Consideration of contact between adjoining areas is required in modelling both the solidification prob- 
lems where the mold is in contact with the casting $[4,5]$ as well as the problems of heat flow in biological tissues [6-8]. Thermal contact can also be used in the problems of damage identification [9].

\section{Mathematical model}

The scheme of the thermal contact between two objects $\Omega_{1}$ and $\Omega_{2}$ that are in contact is shown in Figure 1. Each object has the outer edge $\Gamma_{1}$ or $\Gamma_{2}$ and the internal edge, which is a contact interface where heat transfer takes place. On the fragment $\Gamma_{s}^{0}$ an ideal contact is assumed, while on the fragment $\Gamma_{s}^{g}$ interacting bodies are separated by a gap of the width $h$, which contains a thermally conductive material such as air, water or oil. The width of the gap can vary along $\Gamma_{s}^{g}$.

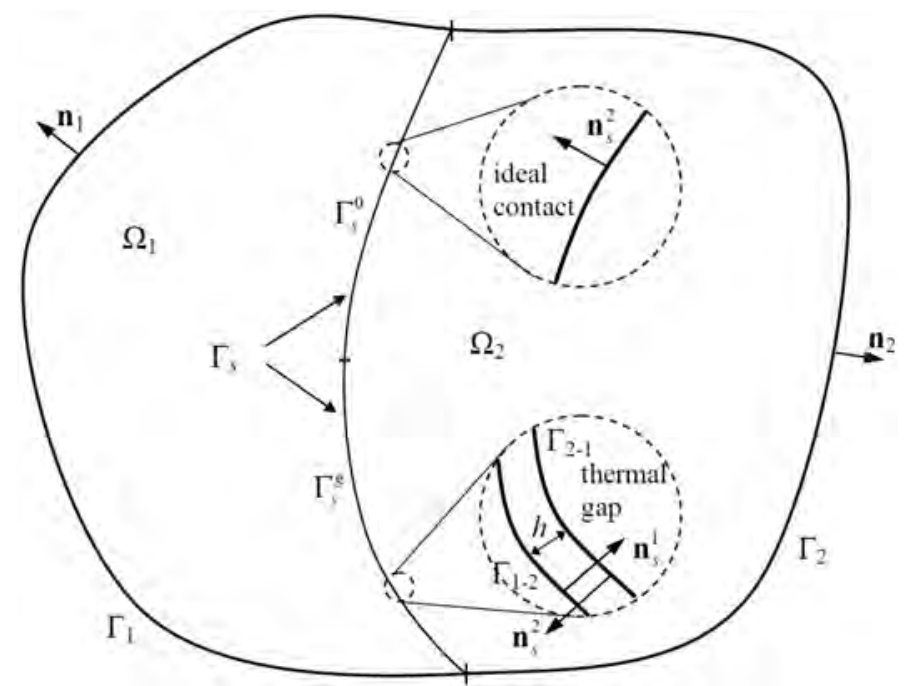

Fig. 1. Scheme of the contact between two flat bodies

The basis of the mathematical model of the problem is the heat conduction equation

$$
\frac{\partial}{\partial x}\left(\lambda_{(i)} \frac{\partial T^{(i)}}{\partial x}\right)+\frac{\partial}{\partial y}\left(\lambda_{(i)} \frac{\partial T^{(i)}}{\partial y}\right)=(c \rho)_{(i)} \frac{\partial T^{(i)}}{\partial t}
$$

where $T^{(i)}[\mathrm{K}]$ is temperature, $\lambda_{(i)}\left[\mathrm{J} \mathrm{m}^{-1} \mathrm{~K}^{-1} \mathrm{~s}^{-1}\right]$ - coefficient of thermal conductivity, $(c \rho)_{(i)}\left[\mathrm{J} \mathrm{m}^{-3} \mathrm{~K}^{-1}\right]$ - heat capacity, $x, y[\mathrm{~m}]$ - spatial coordinates, $t[\mathrm{~s}]$ - time, $i=1,2$ is the subscripts denoting body 1 or 2 .

The initial condition, supplementing the equation (1), takes the following form

$$
T^{(1)}(t=0)=T_{\text {init }}^{(1)}, \quad T^{(2)}(t=0)=T_{\text {init }}^{(2)}
$$


where $T_{\text {init }}^{(1)}, T_{\text {init }}^{(2)}[\mathrm{K}]$ denote known initial temperatures in the regions $\Omega_{1}, \Omega_{2}$ respectively.

At the outer boundaries the following boundary conditions are assumed

$$
-\lambda_{1} \frac{\partial T^{(1)}}{\partial n_{1}}=q_{1}^{*}, \quad-\lambda_{2} \frac{\partial T^{(2)}}{\partial n_{2}}=q_{2}^{*}
$$

where $n_{1}, n_{2}$ are the directions of vectors normal to the boundaries $\Gamma_{1}$ and $\Gamma_{2}$.

At the edge $\Gamma_{s}^{0}$ the following conditions are assumed $[4,5,7]$

$$
\begin{aligned}
-\lambda_{1} \frac{\partial T^{(1)}}{\partial n_{s}^{2}} & =-\lambda_{2} \frac{\partial T^{(2)}}{\partial n_{s}^{2}} \\
T^{(1)} & =T^{(2)}
\end{aligned}
$$

At the edge $\Gamma_{s}^{g}$, where the contact gap is located, the following condition must be fulfilled $[6,8]$

$$
-\lambda_{1} \frac{\partial T^{(1)}}{\partial n_{s}^{1}}=\frac{\lambda_{g}}{h}\left(T^{(1)}-T^{(2)}\right)=\lambda_{2} \frac{\partial T^{(2)}}{\partial n_{s}^{2}}
$$

where $h[\mathrm{~m}]$ denotes the width of the gap, $\lambda_{g}\left[\mathrm{~J} \mathrm{~m}^{-1} \mathrm{~K}^{-1} \mathrm{~s}^{-1}\right]$ is the coefficient of thermal conductivity of the medium in the gap, $T^{(1)}, T^{(2)}[\mathrm{K}]$ are the current temperatures of the bodies 1 and 2 at the contact, while $n_{s}^{1}, n_{s}^{2}$ denote vectors normal to the boundary $\Gamma_{1-2}, \Gamma_{2-1}$, respectively.

At this point of the description of the problem, a more detailed discussion of the introduction of the contact boundary condition is required. Technically speaking, the operation of the modification of the global system of equations is the same as in the case of the third kind of boundary condition, where in equation (5), the heat transfer coefficient $\alpha=\lambda_{g} / h$ and ambient temperature $T_{\infty}=T^{(1)}$ or $T_{\infty}=T^{(2)}$ depending on the modified system of equations. In other words, equation (1) is solved independently in each of the regions and coefficient $\alpha$ in the gap depends on its width and thermal conductivity of the medium, while temperature $T_{\infty}$ is replaced by the boundary temperature of the adjacent region.

\section{Numerical model}

The numerical model is derived from the criterion of the method of weighted residuals [10] in relation to the equation (1). The weighted residual is set to zero

$$
\iint_{\Omega_{i}} w\left(\frac{\partial}{\partial x}\left(\lambda_{(i)} \frac{\partial T^{(i)}}{\partial x}\right)+\frac{\partial}{\partial y}\left(\lambda_{(i)} \frac{\partial T^{(i)}}{\partial y}\right)-(c \rho)_{(i)} \frac{\partial T^{(i)}}{\partial t}\right) d \Omega=0
$$

where $w=w(x, y)$ is the weighting function and $d \Omega=d x d y$. 
The order of equation (6) is decreased using Green's theorem

$$
\iint_{\Omega_{i}}\left(\lambda_{(i)}\left(\frac{\partial w}{\partial x} \frac{\partial T^{(i)}}{\partial x}+\frac{\partial w}{\partial y} \frac{\partial T^{(i)}}{\partial y}\right)+(c \rho)_{(i)} w \frac{\partial T^{(i)}}{\partial t}\right) d \Omega=-\oint_{\Gamma} w q d \Gamma
$$

where $q\left[\mathrm{~J} \mathrm{~s}^{-1} \mathrm{~m}^{-2}\right]$ is the value of heat flux on the external boundaries $\Gamma_{1}, \Gamma_{2}$ or on the internal boundaries $\Gamma_{s}^{0}, \Gamma_{s}^{g}$.

Regions $\Omega_{1}$ and $\Omega_{2}$ are meshed independently. Nodes at $\Gamma_{1-2}$ can have any locations relative to nodes on the $\Gamma_{2-1}$. In the general case, regions $\Omega_{1}$ and $\Omega_{2}$ may be separated by a gap of a curved shape and variable width, filled with a medium characterized by the coefficient of thermal conductivity $\lambda_{g}$. The method of thermal contact modeling in terms of "node to node" approach can be found in [11].

Because the regions $\Omega_{1}, \Omega_{2}$ are separated and the meshes are independent, the equation (1) is solved separately with an appropriate thermal coupling according to the condition (5). In order to generalize the solution procedure, only the condition (5) is used in the model. In the case of perfect contact, when $h=0$, the condition (5) is used with a very small value of $h$. Local heat transfer between the regions $\Omega_{1}$, $\Omega_{2}$ is achieved by thermal coupling of $\Gamma_{1-2}$ and $\Gamma_{2-1}$. The combination of subscripts 1-2 denotes the direction of heat flux through the contact gap from $\Omega_{1}$ to $\Omega_{2}$, while the combination 2-1 denotes direction from $\Omega_{1}$ to $\Omega_{2}$. This coupling makes the equation (1) nonlinear, thus it is necessary to introduce an additional iterative process to achieve high accuracy of numerical calculations.

Temperature and derivatives of temperature with respect to time $t$ and spatial coordinates $x, y$ are approximated using element shape functions, their spatial derivatives and nodal values $T_{j}$

$$
\begin{array}{cc}
T(x, y)=\sum_{j=1}^{3} N_{j}(x, y) T_{j}=\mathbf{N T}^{(e)}, & \frac{\partial T(x, y)}{\partial t}=\sum_{j=1}^{3} N_{j}(x, y) \frac{\partial T_{j}}{\partial t}=\mathbf{N} \dot{\mathbf{T}}^{(e)} \\
\frac{\partial T(x, y)}{\partial x}=\sum_{j=1}^{3} \frac{\partial N_{j}(x, y)}{\partial x} T_{j}=\mathbf{D}_{x} \mathbf{T}^{(e)}, & \frac{\partial T(x, y)}{\partial y}=\sum_{j=1}^{3} \frac{\partial N_{j}(x, y)}{\partial y} T_{j}=\mathbf{D}_{y} \mathbf{T}^{(e)}
\end{array}
$$

where

$$
\begin{gathered}
\mathbf{N}=\left[\begin{array}{lll}
N_{1} & N_{2} & N_{3}
\end{array}\right], \quad \mathbf{D}_{x}=\left[\begin{array}{lll}
\frac{\partial N_{1}}{\partial x} & \frac{\partial N_{2}}{\partial x} & \frac{\partial N_{3}}{\partial x}
\end{array}\right], \quad \mathbf{D}_{y}=\left[\begin{array}{lll}
\frac{\partial N_{1}}{\partial y} & \frac{\partial N_{2}}{\partial y} & \frac{\partial N_{3}}{\partial y}
\end{array}\right] \\
\mathbf{T}^{(e)}=\left\{\begin{array}{l}
T_{1} \\
T_{2} \\
T_{3}
\end{array}\right\}, \quad \dot{\mathbf{T}}^{(e)}=\left\{\begin{array}{l}
\partial T_{1} / \partial t \\
\partial T_{2} / \partial t \\
\partial T_{3} / \partial t
\end{array}\right\}
\end{gathered}
$$


In order to calculate the spatial derivatives of the shape functions, the following formula is used

$$
\left[\begin{array}{l}
\mathbf{D}_{x} \\
\mathbf{D}_{y}
\end{array}\right]=\left(\mathbf{J}^{-1}\right)^{\mathrm{T}}\left[\begin{array}{lll}
\frac{\partial N_{1}}{\partial r} & \frac{\partial N_{2}}{\partial r} & \frac{\partial N_{3}}{\partial r} \\
\frac{\partial N_{1}}{\partial s} & \frac{\partial N_{2}}{\partial s} & \frac{\partial N_{3}}{\partial s}
\end{array}\right]
$$

where $\mathbf{J}$ is the Jacobian matrix of the transformation between coordinates $r, s$ and $x$, $y$ calculated in the following way

$$
\mathbf{J}=\left[\begin{array}{ll}
\frac{\partial x}{\partial r} & \frac{\partial x}{\partial s} \\
\frac{\partial y}{\partial r} & \frac{\partial y}{\partial s}
\end{array}\right]=\left[\begin{array}{ll}
\sum_{j=1}^{3} \frac{\partial N_{j}}{\partial r} x_{j} & \sum_{j=1}^{3} \frac{\partial N_{j}}{\partial s} x_{j} \\
\sum_{j=1}^{3} \frac{\partial N_{j}}{\partial r} y_{j} & \sum_{j=1}^{3} \frac{\partial N_{j}}{\partial s} y_{j}
\end{array}\right]
$$

where $x_{j}, y_{j}$ denotes nodal coordinates.

Relations (8) used with the formula (7) makes it possible to obtain local energy equation

$$
\lambda^{(e)} \iint_{\Omega^{(e)}}\left(\mathbf{D}_{x}^{\mathrm{T}} \mathbf{D}_{x}+\mathbf{D}_{y}^{\mathrm{T}} \mathbf{D}_{y}\right) d \Omega \mathbf{T}^{(e)}+(c \rho)^{(e)} \iint_{\Omega^{(e)}} \mathbf{N}^{\mathrm{T}} \mathbf{N} d \Omega \dot{\mathbf{T}}^{(e)}=-\oint_{\Gamma^{(e)}} \mathbf{N} q d \Gamma
$$

Assuming the following relations

$$
\begin{gathered}
\mathbf{K}^{(e)}=\lambda^{(e)} \iint_{\Omega^{(e)}}\left(\mathbf{D}_{x}^{\mathrm{T}} \mathbf{D}_{x}+\mathbf{D}_{y}^{\mathrm{T}} \mathbf{D}_{y}\right) d \Omega \\
\mathbf{M}^{(e)}=(c \rho)^{(e)} \iint_{\Omega^{(e)}} \mathbf{N}^{\mathrm{T}} \mathbf{N} d \Omega \\
\mathbf{B}^{(e)}=-\oint_{\Gamma^{(e)}} \mathbf{N} q d \Gamma
\end{gathered}
$$

where $\mathbf{K}^{(e)}$ is the thermal conductivity matrix, $\mathbf{M}^{(e)}$ - heat capacity matrix, $\mathbf{B}^{(e)}$ - right-hand side vector, equation (13) can be written as

$$
\mathbf{K}^{(e)} \mathbf{T}^{(e)}+\mathbf{M}^{(e)} \dot{\mathbf{T}}^{(e)}=\mathbf{B}^{(e)}
$$

The operation of aggregation leads to the following global equation

$$
\mathbf{K T}+\mathbf{M} \dot{\mathbf{T}}=\mathbf{B}
$$


Derivative of the temperature $\dot{\mathbf{T}}$ with respect to time is approximated by the following linear differential scheme

$$
\dot{\mathbf{T}}=\frac{\mathbf{T}^{f+1}-\mathbf{T}^{f}}{\Delta t}
$$

where $f$ is the time level, $\Delta t$ [s] means time step between level $f$ and $f+1$. Using scheme (19) in equation (18) the following expression is obtained

$$
\mathbf{K} \mathbf{T}^{f+1}+\mathbf{M} \frac{\mathbf{T}^{f+1}-\mathbf{T}^{f}}{\Delta t}=\mathbf{B}
$$

After arranging the above equation with respect to the time level, the global FEM equation discretized using the implicit Euler method is obtained

$$
\left(\mathbf{K}+\frac{1}{\Delta t} \mathbf{M}\right) \mathbf{T}^{f+1}=\mathbf{B}+\frac{1}{\Delta t} \mathbf{M T}^{f}
$$

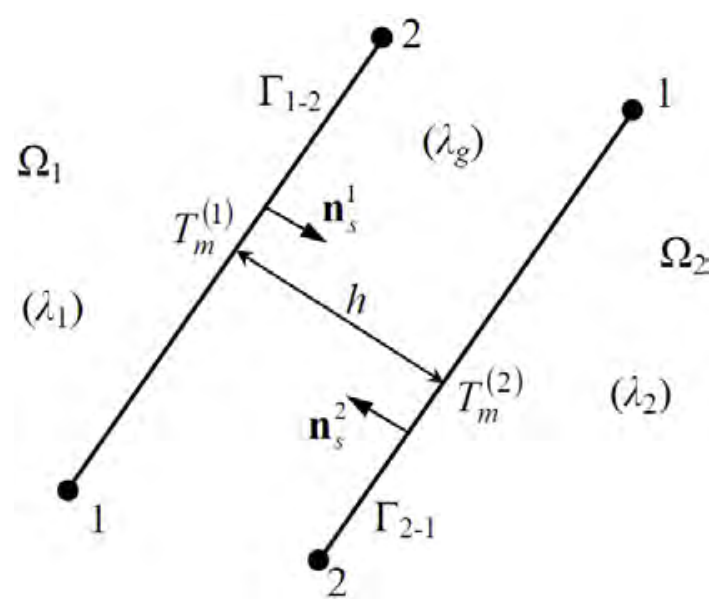

Fig. 2. Thermal contact between two elements

For the region $\Omega_{1}$ on the boundary $\Gamma_{1-2}$ (Fig. 2) the following condition must be satisfied

$$
-\lambda_{1} \frac{\partial T_{m}^{(1)}}{\partial n_{s}^{1}}=\frac{\lambda_{g}}{h}\left(T_{m}^{(1)}-T_{m}^{(2)}\right)
$$

while for the region $\Omega_{2}$ on the $\Gamma_{2-1}$

$$
-\lambda_{2} \frac{\partial T_{m}^{(2)}}{\partial n_{s}^{2}}=\frac{\lambda_{g}}{h}\left(T_{m}^{(2)}-T_{m}^{(1)}\right)
$$


At the fragment $\Gamma_{1-2}$ the position of the central point is calculated and then the direction normal to $\Gamma_{2-1}$ is estimated to find the local width of the gap and the temperature $T_{m}^{(2)}$ at the fragment $\Gamma_{2-1}$. Then the modification of the set of equations using condition (24) for region $\Omega_{1}$ is made. The same procedure is carried out for the $\Omega_{2}$. Heat flux at the edge between nodes 1-2 is integrated as follows

$$
\int_{\Gamma_{1-2}} \mathbf{N} \frac{\lambda_{g}}{h}\left(T_{m}^{(1)}-T_{m}^{(2)}\right) d \Gamma=\frac{\lambda_{g}}{h} \int_{\Gamma_{1-2}} \mathbf{N}^{\mathrm{T}} \mathbf{N} d \Gamma \mathbf{T}^{(e)}-\frac{\lambda_{g}}{h} T_{m}^{(2)} \int_{\Gamma_{2-1}} \mathbf{N} d \Gamma
$$

The first integral on the right side of expression (24) modifies local matrix $\mathbf{K}^{(e)}$ while the second one modifies vector $\mathbf{B}^{(e)}$. In an analogous manner, the influence of the region $\Omega_{1}$ on the region $\Omega_{2}$ is considered

$$
\int_{\Gamma_{2-1}} \mathbf{N} \frac{\lambda_{g}}{h}\left(T_{m}^{(2)}-T_{m}^{(1)}\right) d \Gamma=\frac{\lambda_{g}}{h} \int_{\Gamma_{2-1}} \mathbf{N}^{\mathrm{T}} \mathbf{N} d \Gamma \mathbf{T}^{(e)}-\frac{\lambda_{g}}{h} T_{m}^{(1)} \int_{\Gamma_{1-2}} \mathbf{N} d \Gamma
$$

It should be emphasized that the values of $T_{m}^{(1)}, T_{m}^{(2)}$ on the opposite sides of the gap should be calculated using an additional iterative procedure in each time step to achieve desired accuracy. Such an approach extends the time of calculations but allows for the economical use of the computer's memory.

\section{Conclusions}

The presented mathematical and numerical models of the thermal contact problem enables computer implementation of the in-home solver. The presented approach based on the separated meshes allows one to solve complex problems, because the current operational memory usage is limited to one mesh. The temperature in the entire region is determined on the basis of an iterative process.

\section{References}

[1] Cooper M.G., Mikic B.B., Yovanovich M.M., Thermal contact conductance, Int. J. Heat Mass Transf. 1969, 12, 279-300.

[2] Schneider G.E., Strong A.B., Yovanovich M.M., Transient thermal response to two bodies communicating through a small circular contact area, Int. J. Heat Mass Transf. 1977, 20, 301-308.

[3] Longa W., Krzepnięcie odlewów w formach piaskowych, Śląsk, Katowice 1973.

[4] Majchrzak E., Mendakiewicz J., Piasecka-Belkhayat A., Algorithm of the mould thermal parameters identification in the system casting-mould-environment, J. Mater. Process. Technol. 2005, 164, 1544-1549.

[5] Mendakiewicz J., Identification of solidification process parameters, Computer Assisted Mechanics and Engineering Sciences (CAMES) 2010, 17(1), 59-73. 
[6] Ciesielski M., Mochnacki B., Numerical simulation of the heating process in the domain of tissue insulated by protective clothing, J. Appl. Math. Comput. Mech. 2014, 13(2), 13-20.

[7] Mochnacki B., Piasecka-Belkhayat A., Numerical modeling of skin tissue heating using the interval finite difference method, Mol. Cell. Biomech. 2013, 10(3), 233-244.

[8] Mochnacki B., Majchrzak E., Duda M., Simplified model of thermal interactions between environment protective clothing and skin tissue, J. Appl. Math. Comput. Mech. 2014, 13(4), 91-96.

[9] Ziopaja K., Pozorski Z., Garstecki A., Damage detection using thermal experiments and wavelet transformation, Inverse Problems in Science and Engineering 2011, 19(1), 127-153.

[10] Grandin H., Fundamentals of the Finite Element Method, Waveland Press 1991.

[11] Sczygiol N., Modelowanie numeryczne zjawisk termomechanicznych w krzepnącym odlewie i formie odlewniczej, Wydawnictwo Politechniki Częstochowskiej, Częstochowa 2000. 